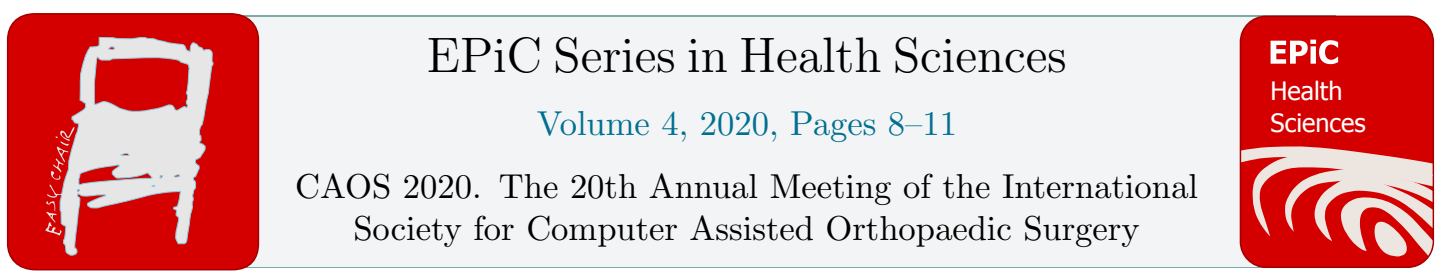

\title{
Combining sensor and robotic technologies to achieve a well balanced total knee arthroplasty while avoiding any soft tissue releases
}

\author{
Julien Bardou-Jaquet \\ Clinique Tivoli-Ducos, France \\ jbardoujacquet@me.com
}

\section{Introduction}

Achieving a balanced total knee throughout the entire range of motion leads to improved patient reported outcomes and satisfaction (Hasegawa et al., 2018; Golladay et al., 2019). Sensor-assisted technology allows the surgeon to quantitatively assess and address imbalance through either soft tissue releases or bone recuts (Meneghini et al., 2016; Gustke et al., 2017). However, balancing through soft tissue releases leads to unpredictable gap increments and frequent early over-releases (Kwak et al., 2016).

The primary objective of this study was to demonstrate the ability to achieve a quantitatively balanced knee by combining two technologies, namely robotic arm and intra-operative load sensors, while avoiding any soft tissue corrections.

\section{Methods}

This study leveraged the ability of a robotic platform (MAKO, Stryker) to control and adjust, if necessary, the implant positioning while maintaining this positioning in a safe zone regarding the femoral and tibial component alignment (mechanical alignement). Intra-operative feedback from load sensors (VERASENSE, Orthosensor) was thereby used as a precise diagnostic tool.

During a consecutive and prospective series of 29 robotic arm total knee arthroplasties, intraoperative load sensors were used following the initial bone resections to quantitatively assess the knee's state of balance through the range of motion with trial components in place. Load measurements were taken at 10 and 90 degrees of knee flexion. Based on previous literature (Gustke et al., 2014) a balanced knee is defined as having a mediolateral load difference below 15 pounds forces (lbf) through the range of motion, with an absolute load magnitude per compartment above $5 \mathrm{lbf}$ and not exceeding $45 \mathrm{lbf}$.

The initial load numbers were recorded as well as the number and type of subsequent corrections needed to achieve quantitative balance. Bony recuts are made $0,5 \mathrm{MM}$ by $0,5 \mathrm{MM}$ if necessary on tibial or femoral side according to load numbers. 
A

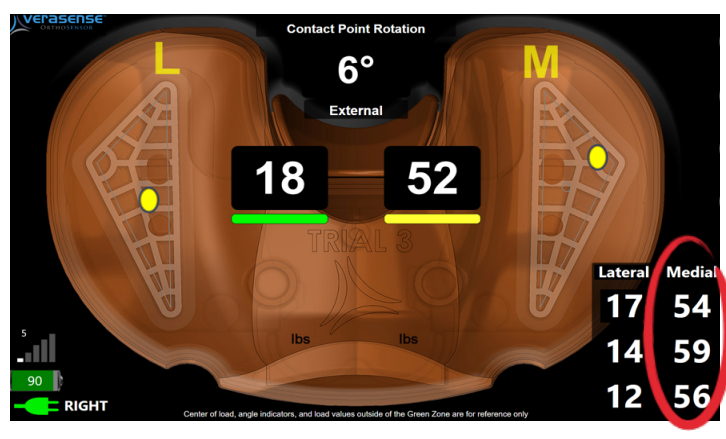

C

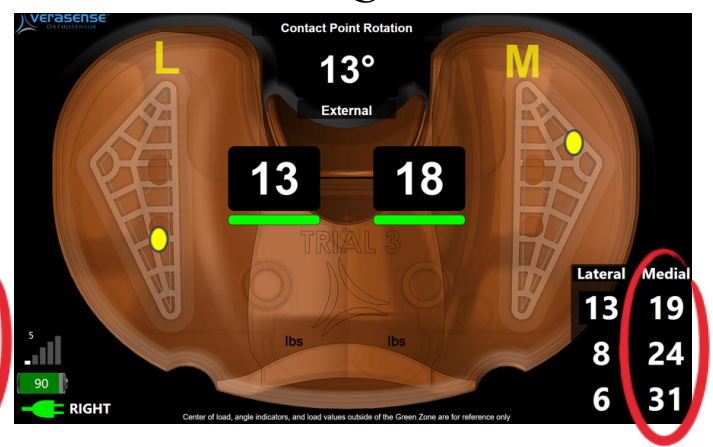

B
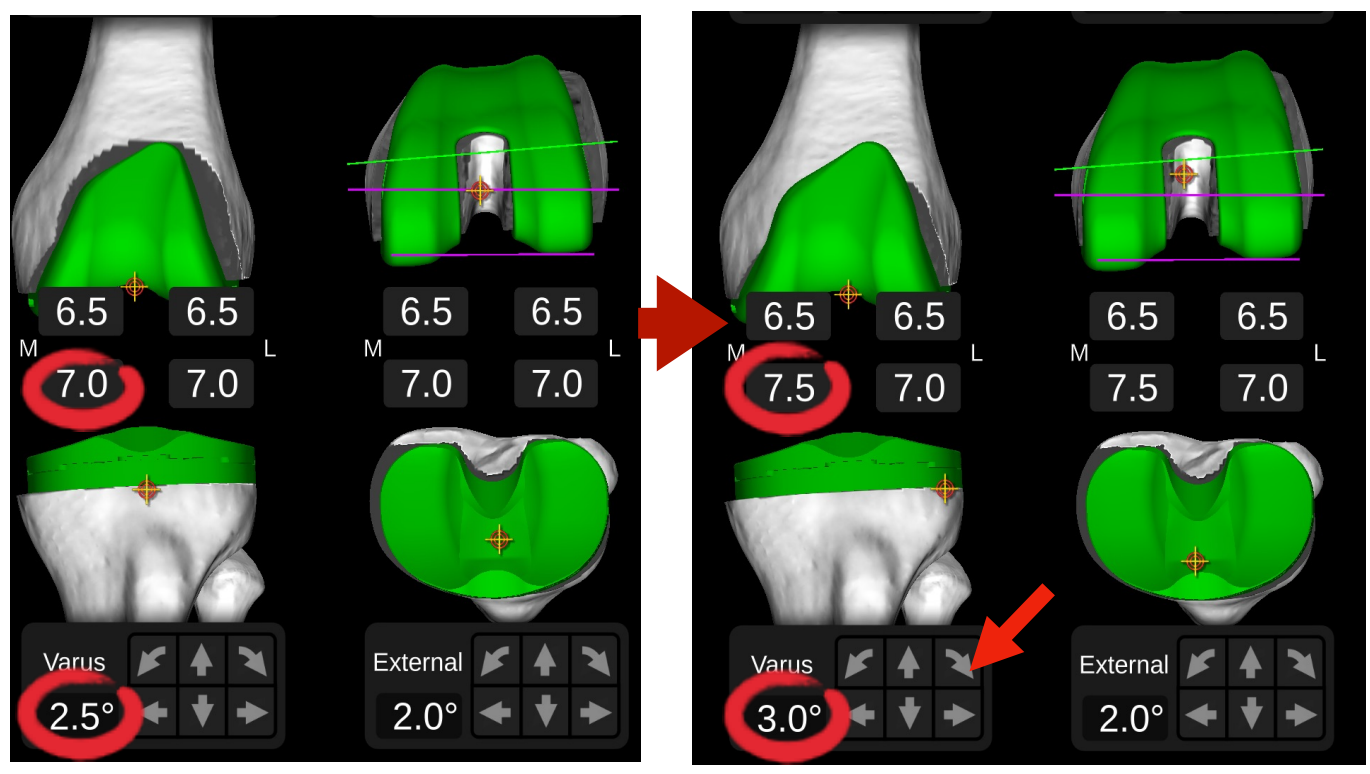

Figure 1: A - The knee is tight in flexion and extension; B - The center of rotation of tibial cut in the frontal plan is move on lateral edge, 0,5 degree of varus is added so we cut $0,5 \mathrm{MM}$ more on the medial side of the tibia; $\mathrm{C}$ - The control with load sensor shows a perfect balanced knee.

\section{Results}

Of the 29 robotics cases, only $12(41 \%)$ were well-balanced after the initial bone cuts (mechanical alignment by measured resection). Another two cases were too loose and required an increase in the polyethylene thickness size of two millimeters to achieve a well-balanced knee without further bone resection. In 14 cases, a bone recut was required to balance the knee. More specifically, four cases required a recut of the femur, ten cases required a recut of the tibia. Eventually, one case was left unbalanced in flexion with a mediolateral load differential of $20 \mathrm{lbf}$. It should be noted explicitly that no soft tissue releases were done for any of the 29 cases. At the end, all 29 knees were considered well 


\begin{tabular}{|c|c|c|c|c|c|c|c|c|c|c|c|c|c|c|c|c|c|c|c|c|c|c|c|}
\hline \multirow[t]{3}{*}{$D$} & \multirow[t]{3}{*}{$\sigma$} & \multicolumn{4}{|c|}{$\Omega$} & \multirow[t]{2}{*}{$\theta$} & \multicolumn{4}{|c|}{$x$} & \multicolumn{2}{|c|}{ x } & \multicolumn{2}{|c|}{$a$} & \multicolumn{2}{|c|}{ \pm} & \multicolumn{4}{|c|}{-} & \multirow[t]{2}{*}{-} & \multirow[t]{2}{*}{$\pi$} & \multirow[t]{2}{*}{ 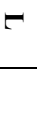 } \\
\hline & & \multicolumn{2}{|c|}{ 。 } & \multicolumn{2}{|c|}{$\stackrel{0}{0}$} & & \multicolumn{2}{|c|}{ 。 } & \multicolumn{2}{|c|}{ ๕̊ } & \multirow[b]{2}{*}{3} & \multirow[b]{2}{*}{$\Gamma$} & \multirow[b]{2}{*}{3} & \multirow[b]{2}{*}{$r$} & \multirow[b]{2}{*}{3} & \multirow[b]{2}{*}{$\sigma$} & \multicolumn{2}{|c|}{$\bar{\theta}_{0}$} & \multicolumn{2}{|c|}{$\stackrel{0}{0}$} & & & \\
\hline & & 3 & $F$ & 3 & $\Gamma$ & & 3 & $\sigma$ & 3 & $r$ & & & & & & & 3 & $\sigma$ & 3 & $F$ & & & \\
\hline$\vec{u}$ & $a$ & $u$ & $\$$ & N & $\bar{\omega}$ & & & & & & & & - & - & & & $\underset{v}{w}$ & $\underset{\omega}{w}$ & 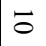 & $\vec{u}$ & $\vec{v}$ & 0 & $\Xi$ \\
\hline$\exists$ & N & N & $\simeq$ & $\stackrel{\sim}{\omega}$ & 0 & $N$ & & & & & & & & & \pm & 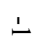 & $\tilde{u}$ & $\vec{u}$ & $\sim$ & $\tilde{u}$ & $\vec{u}$ & 0 & $\Xi$ \\
\hline$\vec{\nabla}$ & 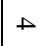 & $b$ & $\vec{a}$ & \pm & $\checkmark$ & & & & & & $\bar{i}_{n}$ & $i_{i}$ & & & & & $\stackrel{\infty}{\infty}$ & o & $\tilde{\alpha}$ & $\sigma$ & $\vec{\infty}$ & 0 & $=$ \\
\hline$\vec{w}$ & 0 & 0 & $\tilde{\sigma}$ & $\bar{N}$ & $\stackrel{N}{A}$ & N & bे & $a$ & $\vec{\infty}$ & 0 & in & - & & & & & No & $\checkmark$ & $\tilde{V}$ & $\infty$ & $\vec{\infty}$ & - & $=$ \\
\hline$\vec{v}$ & 0 & תू & $\vec{\sigma}$ & $\infty$ & $\bar{\sigma}$ & $N$ & & & & & - & & & & & & $\bar{N}$ & N & $\overrightarrow{0}$ & $\not$ & $\vec{\infty}$ & 0 & ஏ \\
\hline$\varpi$ & 0 & ur & 岕 & $\checkmark$ & $\infty$ & & & & & & $i_{i r}$ & & & & & & $\overrightarrow{0}$ & ন & $\sigma$ & $\vec{A}$ & $\infty$ & 0 & 0 \\
\hline$\vec{\sigma}$ & $\bar{a}$ & $\stackrel{\vec{\infty}}{ }$ & $\nabla$ & $w_{\sigma}$ & $\tilde{o}$ & & & & & & in & & & & & & $\bar{u}$ & N & $\sigma$ & $\tilde{\perp}$ & $\overrightarrow{0}$ & $\omega$ & 6 \\
\hline$\ddot{\infty}$ & 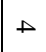 & $\ddot{t}$ & $\exists$ & ŏ & $\bar{N}$ & & & & & & in & & & & & & $\sigma$ & $\bar{\omega}$ & $\ddot{o}$ & $a$ & $\vec{\infty}$ & $\therefore$ & 0 \\
\hline$\vec{\omega}$ & $\checkmark$ & $\underline{w}$ & \pm & $\underset{w}{w}$ & $\underset{v}{w}$ & & & & & & & $i_{1}$ & & & & & N & $\sigma$ & 二 & $\vec{\perp}$ & $\vec{u}$ & ur & 0 \\
\hline$\vec{\exists}$ & $\infty$ & $\stackrel{2}{2}$ & $\underset{\infty}{\infty}$ & รั & $\underline{\omega}$ & & & & & & in & & & & & & $\bar{N}$ & $\checkmark$ & $\bar{N}$ & $\checkmark$ & $\vec{y}$ & $\omega$ & 0 \\
\hline$\vec{\nabla}$ & $a$ & $\vec{\infty}$ & $\sigma$ & $\mathrm{N}$ & N & & & & & & & & in & & & & N & 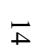 & $\not{A}$ & $\infty$ & $\bar{\infty}$ & 0 & 6 \\
\hline$\vec{a}$ & 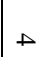 & $\underset{\nexists}{\omega}$ & $=$ & 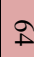 & $\infty$ & & & & & & - & & & & & & 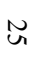 & $\omega_{\infty}$ & $\vec{\perp}$ & N & $\vec{\infty}$ & - & 0 \\
\hline$\vec{\sigma}$ & - & $\bar{\sigma}$ & $\sim$ & $\infty$ & N & & & & & & & ir & & & & & 0 & 6 & $\checkmark$ & $\tilde{\sigma}$ & $\vec{\infty}$ & 0 & 0 \\
\hline$\ddot{\infty}$ & $\checkmark$ & $\mathcal{N}$ & $\mp$ & $\omega$ & $a$ & & & & & & & & & & ir & & $\widetilde{O}$ & 二 & $\vec{u}$ & $v$ & $\Phi$ & 0 & 0 \\
\hline
\end{tabular}

Table 1: A - Initial Coronal Limb Alignment HKA $\left({ }^{\circ}\right)$; B - Fixed Flexion (flessum) $\left({ }^{\circ}\right)$; C - Sensor Trial(lbf); D - PE Increase (mm); E - Sensor Trial (lbf); F - Tibial recut (mm); G - Femoral distal recut (mm); H -

Posterior condyle recut (mm); I - Sensor Final (lbf); J - Final Coronal Limb Alignment HKA; K - Final Fixed Flexion; L - PE Final (mm).

balanced in extension and all but six $(79 \%)$ at $90^{\circ}$ of flexion. For these six cases with balance issue at $90^{\circ}$ of flexion, absolute load magnitude in both compartments was below $45 \mathrm{lbf}$ and above 5lbf, though the mediolateral load differential was between $15 \mathrm{lbf}$ and $30 \mathrm{lbf}$. 


\section{Discussion}

Based on a preliminary series, this work demonstrates the opportunity of combining multiple technologies to achieve a quantitatively balanced knee through the range of motion. In contrast to previous work reported in literature (Gordon, 2019), this study emphasizes the opportunity to achieve a balanced joint while only relying on patient-specific bone recuts guided by intra-operative load sensor readings, thus sparing the soft tissues surrounding the knee joint.

All data collected will allow us to make the procedure reproducible, predictable and thus to enhance concepts of ligament balancing in total knee arthroplasty and perhaps improve patient satisfaction (Hasegawa et al., 2018).

\section{References}

Hasegawa, M., et al., Factors Contributing to Patient Satisfaction and Expectations following Computer-Assisted Total Knee Arthroplasty. J Knee Surg, 2018. 31(5): p. 448-452.

Golladay, G.J., et al., Are Patients More Satisfied With a Balanced Total Knee Arthroplasty? J Arthroplasty, 2019. 34(7S): p. S195-S200.

Meneghini, R.M., et al., Can Intraoperative Sensors Determine the "Target" Ligament Balance? Early Outcomes in Total Knee Arthroplasty. J Arthroplasty, 2016. 31(10): p. 2181-7.

Gustke, K.A., et al., A Targeted Approach to Ligament Balancing Using Kinetic Sensors. J Arthroplasty, 2017. 32(7): p. 2127-2132.

Kwak, D.S., et al., The pie-crusting technique using a blade knife for medial collateral ligament release is unreliable in varus total knee arthroplasty. Knee Surg Sports Traumatol Arthrosc, 2016. 24(1): p. 188-94.

Gustke, K.A., et al., A new method for defining balance: promising short-term clinical outcomes of sensor-guided TKA. J Arthroplasty, 2014. 29(5): p. 955-60.

Gordon, A. Combining Multiple Technologies to Achieve Optimized Surgical Outcome in Total Knee Arthroplasty. 2019. European Knee Society, Open Meeting, Valencia, Spain. 